\section{PRÁCTICAS DEL PERSONAL DE SALUD RELACIONADAS CON LAS MEDIDAS DE PREVENCIÓN DE LA TUBERCULOSIS}

\author{
PRACTICES OF THE HEALTH CARE \\ PERSONNEL IN RELATION TO TUBERCULOSIS \\ PREVENTION MEASURES
}

\author{
Manuel A. Virú-Loza 1,2,a
}

En los medios hospitalarios peruanos hay un riesgo muy elevado de adquirir tuberculosis ${ }^{(1)}$, que se puede extender a otros niveles de atención. Por ello, se han emitido recomendaciones y guías nacionales e internacionales para la prevención y control de tuberculosis. Sin embargo, un tema crítico aún por resolver en nuestro país son los problemas de implementación de estas recomendaciones y guías ${ }^{(2)}$; en este contexto, es importante hacer que el personal adopte las medidas de protección establecidas.

Los profesionales de salud conocen las formas de contagio de tuberculosis y reconocen la existencia de medidas de prevención básicas como el uso de mascarillas faciales; sin embargo, esto no asegura que acaten estas medidas ${ }^{(1,3)}$. Este problema no solo se debe a disponibilidad de recursos que pueda utilizar el personal de salud para protegerse sino que también podría implicar la poca disposición a acatar, por diversos motivos, las medidas establecidas.

Actitudes como la disconformidad que expresan algunos profesionales de la salud con respecto al uso y a las características de las mascarillas faciales ${ }^{(3,4)}$, teniendo en cuenta la evidencia que sugeriría que no ocurren variaciones fisiológicas o subjetivas significativas producto del uso de máscaras quirúrgicas durante una hora ${ }^{(5)}$, serían una muestra de poca colaboración al cumplimiento de las normas. Sin embargo, este no es el único argumento. Otros más preocupantes tratan sobre que el uso de máscaras faciales podría hacer que el paciente se sienta discriminado; incluso están los profesionales que indican que es una medida poco útil puesto que ellos llevan varios años en el mismo establecimiento y no han contraído tuberculosis ni

\footnotetext{
Universidad Nacional Mayor de San Marcos. Lima, Perú.

Asociación para el Desarrollo de la Investigación Estudiantil en Ciencias de la Salud (ADIECS-UNMSM). Lima, Perú.

a Estudiante de Medicina

Recibido: 07-10-12 Aprobado: 31-10-12
}

Citar como: Virú-Loza MA. Prácticas del personal de salud relacionadas con las medidas de prevención de la tuberculosis. Rev Peru Med Exp Salud Publica. 2012;29(4):582. han visto que algún compañero de trabajo la haya contraído.

Estos comportamientos y actitudes hacia las normas preventivas y de control son observados por los estudiantes e internos de las ciencias de la salud, quienes están en un proceso de adquisición no solo de conocimientos sino también de experiencias. Como bien se puede deducir, los ejemplos inadecuados de algunos profesionales pueden afectar seriamente las acciones futuras que tomen los estudiantes expuestos a este tipo de influencia, cayendo así en un círculo vicioso.

Por tanto, se recomienda estudiar las actitudes y prácticas relacionadas a las medidas de prevención y control de tuberculosis en el personal de salud, así como también las estrategias que mejoren la aceptación de los profesionales de la salud hacia estas medidas. Al mismo tiempo, es necesario ser más estrictos acerca del uso de medidas de protección. Se debe incluir sanciones severas para contribuir a cambiar el comportamiento de aquellos profesionales que se resistan a aplicar medidas de protección. Todo esto no solo con la finalidad de prevenir la tuberculosis en el personal de salud sino también para evitar que los estudiantes que asisten a los diferentes centros de salud observen y repitan malas prácticas.

\section{REFERENCIAS BIBLIOGRÁFICAS}

1. Bonifacio N, Saito M, Gilman RH, Leung F, Cordova Chavez $\mathrm{N}$, Chacaltana Huarcaya J, et al. High risk for tuberculosis in hospital physicians, Peru. Emerg Infect Dis. 2002;8(7):747-8.

2. Cabezas C. Tuberculosis en personal y estudiantes de salud: un tema pendiente para los servicios de salud y la universidad. Rev Peru Med Exp Salud Publica. 2012;29(2):179-80.

3. Huaroto L, Espinoza MM. Recomendaciones para el control de la transmisión de la tuberculosis en los hospitales. Rev Peru Med Exp Salud Publica. 2009;26(3):364-9.

4. Baig AS, Knapp C, Eagan AE, Radonovich LJ Jr. Health care workers' views about respirator use and features that should be included in the next generation of respirators. Am J Infect Control. 2010;38(1):18-25.

5. Roberge RJ, Kim JH, Benson SM. Absence of consequential changes in physiological, thermal and subjective responses from wearing a surgical mask. Respir Physiol Neurobiol. 2012;181(1):29-35.

Correspondencia: Manuel André Virú-Loza

Dirección: Jr. Piedra Bigua 2241-a, San Juan de Lurigancho. Lima 36, Perú.

Telefono: (511) 3043417

Correo electrónico: andreviru@hotmail.com 